Acta Crystallographica Section C

Crystal Structure

Communications

ISSN 0108-2701

\title{
1,1,3,3-Tetramethylguanidinium dihydrogenorthophosphate
}

\section{A. Criado, ${ }^{\mathrm{a} *}$ M. J. Diánez, ${ }^{\mathrm{a}}$ S. Pérez-Garrido, ${ }^{\mathrm{a}}$ I. M. L. Fernandes, ${ }^{b}$ M. Belsley ${ }^{b}$ and E. de Matos Gomes ${ }^{b}$}

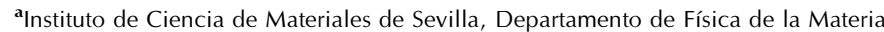
Condensada, CSIC - Universidad de Sevilla, Apartado 1065, 41080 Sevilla, Spain, and ${ }^{\mathbf{b}}$ Departamento de Física, Universidade do Minho, 4709 Braga, Portugal Correspondence e-mail: criado@cica.es

Received 2 March 2000

Accepted 5 April 2000

In the title compound, $\mathrm{C}_{5} \mathrm{H}_{14} \mathrm{~N}_{3}^{+} \cdot \mathrm{H}_{2} \mathrm{PO}_{4}^{-}$, the cation has a central guanidinium fragment with a planar geometry, as expected for a central $\mathrm{Csp}^{2}$ atom with a small charge delocalization along the three $\mathrm{C}-\mathrm{N}$ bonds. The crystal packing is governed by hydrogen bonds so that the phosphate anions are linked head to tail, forming chains running parallel to the $c$ direction. These chains in turn are interconnected by hydrogen bonds to intermediate tetramethylguanidinium cations forming hydrogen-bonded molecular layers stacked parallel to the $b c$ crystal planes.

\section{Comment}

Inorganic salts of phosphoric acids form compounds that exhibit a wealth of interesting physical properties such as ferroelectricity and non-linear optical phenomena like second harmonic generation; a classical example is potassium dihydrogen orthophosphate (KDP) (Rafhkovich, 1991). A general synthetic route to obtain organo-dihydrogen orthophosphate crystals has been detailed (Masse \& Zyss, 1991). In all these compounds, there is an inorganic subnetwork formed by the dihydrogen orthophosphate anions $\left(\mathrm{H}_{2} \mathrm{PO}_{4}\right)_{n}$. When the organic species are strongly dipolar, the anion sublattice is

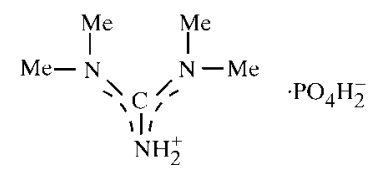

(I)

organized in a polar structure; examples are L-argininium dihydrogen orthophosphate monohydrate (Aoki et al., 1971), 2amino-5-nitropyridinium dihydrogen orthophosphate (Kotler et al., 1992) and sarcosine dihydrogen orthophosphate (Averbuch-Pouchot et al., 1988). In the case of a weakly dipolar organic species such as glycine, the anion sublattice will organize in a nonpolar structure (Averbuch-Pouchot et al., 1988). As part of a project to study new compounds with potentially interesting optical and dielectric properties, we have synthesized the title compound, (I). Similar to aminoguanidinium dihydrogen orthophosphate (Adams, 1977), we report here its crystal structure, as determined by singlecrystal X-ray diffraction.

The compound crystallizes in a centrosymmetric space group; consequently, no non-linear optical effects are observed. Differential scanning calorimetry measurements performed from 93 to $673 \mathrm{~K}$ did not show any phase transition. The melting point occurs at about $493 \mathrm{~K}$, followed by decomposition.

The geometry of the guanidinium group in (I) is planar, as expected for $s p^{2}$ hybridization of the central $\mathrm{C}$ atom (Fig. 1). The $\pi$ delocalization along the three $\mathrm{C}-\mathrm{N}$ bonds gives rise to

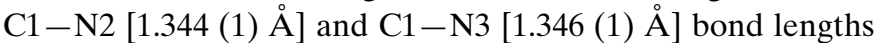
larger than the value expected for a Csp $p^{2}=\mathrm{N}$ bond $(1.295 \AA)$ and close to the expected value for a delocalized $\mathrm{C}=\mathrm{N}$ double

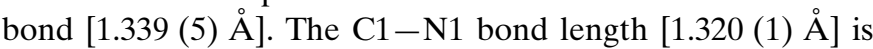
somewhat shorter and compares well with the average value for the guanidinium cation (1.321 $\AA$ ) (Allen et al., 1987). The larger value for the $\mathrm{C} 1-\mathrm{N} 2$ and $\mathrm{C} 1-\mathrm{N} 3$ bond lengths must be ascribed to the methyl substitution which makes the three bonds non-equivalent. Indeed, simple molecular-orbital semiempirical calculations (extended Hückel) give different atomic charges on $\mathrm{N} 1(-0.376 \mathrm{e})$ and $\mathrm{N} 2$ and $\mathrm{N} 3(-0.590 \mathrm{e})$. As may be expected, the two $\mathrm{P}-\mathrm{O}$ distances for the $\mathrm{OH}$ groups are significantly longer than the other two $\mathrm{P}-\mathrm{O}$ distances.

The basis of the molecular engineering interest in these salts is the obtention of structures with potential physical properties as a result of the hydrogen-bond crystal network, which tends to reinforce the properties exhibited by the isolated molecule by arranging them as linear or layered molecular patterns.

In our case the hydrogen bonds also give rise to an interesting arrangement, which is best understood with the aid of the diagram corresponding to the crystal structure viewed perpendicular to the $a b$ plane (Fig. 2). On one hand, each phosphate ion is connected by two hydrogen bonds to each phosphate ion related to it by a $c$ glide plane with both positive and negative fractional $c$ translations. Given that the central $\mathrm{P}$ atoms lie at a very close distance to the glide planes $(0.32 \AA)$, it results in approximately linear phosphate chains parallel to the $c$ direction. On the other hand, each tetramethylguanidinium ion is hydrogen-bonded to two phosphate ions related to each other by an inversion centre and belonging to different chains, giving rise to a framework of molecules
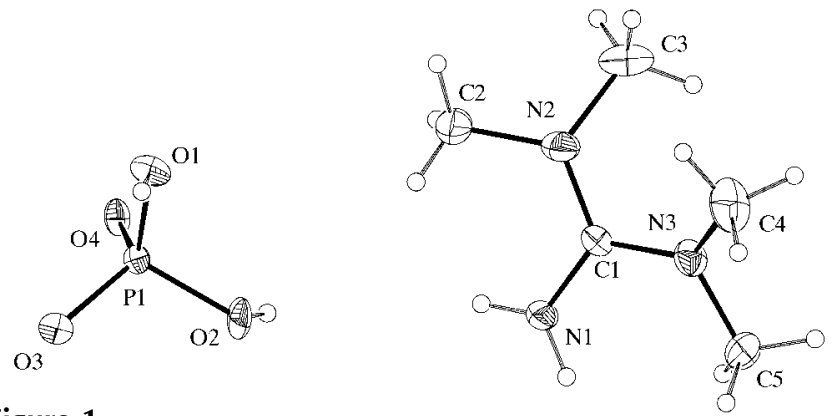

Figure 1

Structure of (I) showing 30\% probability displacement ellipsoids. 
connected by hydrogen bonds in the form of layers parallel to the $b c$ crystal planes and stacked according to the $a$ lattice translation period. Besides the above, two $\mathrm{C}-\mathrm{H} \cdots \mathrm{O}$ contacts interrelating molecules within the same layer and not depicted in the diagram have been detected.

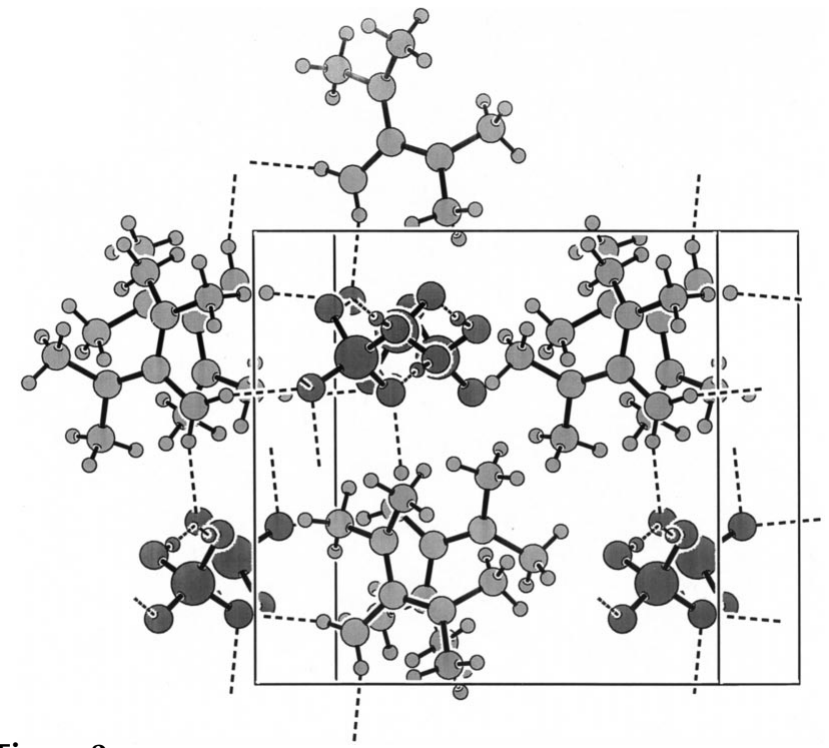

Figure 2

The molecular packing viewed along an axis perpendicular to the $a b$ plane showing the hydrogen bonding.

\section{Experimental}

The title compound was prepared by mixing equimolar portions of two reagents: 1,1,3,3-tetramethylguanidine (99\%) and phosphoric acid $(85 \%)$ in a $1: 1$ solution of ethanol and water at room temperature. Good quality, colourless single crystals of prism habit were grown from the solution by slow evaporation, one of which was selected and used for the X-ray analysis.

\section{Crystal data}

$\mathrm{C}_{5} \mathrm{H}_{14} \mathrm{~N}_{3}^{+} \cdot \mathrm{H}_{2} \mathrm{PO}_{4}^{-}$

$M_{r}=213.18$

Monoclinic, $P 2_{1} / c$

$a=11.225(3) \AA$

$b=10.951(1) \AA$

$c=8.430(2) \AA$

$\beta=103.50(1)^{\circ}$

$V=1007.6(4) \AA^{3}$

$Z=4$

$D_{x}=1.405 \mathrm{Mg} \mathrm{m}^{-3}$

$D_{m}=1.40 \mathrm{Mg} \mathrm{m}^{-3}$

\author{
$D_{m}$ measured by flotation in \\ bromobenzene and acetone \\ Mo $K \alpha$ radiation \\ Cell parameters from 25 \\ reflections \\ $\theta=7-12^{\circ}$ \\ $\mu=0.264 \mathrm{~mm}^{-1}$ \\ $T=293$ (2) K \\ Prism, colourless \\ $0.80 \times 0.50 \times 0.40 \mathrm{~mm}$
}

\section{Data collection}

Enraf-Nonius CAD-4 diffractometer

$\omega-2 \theta$ scans

3777 measured reflections

3777 independent reflections

3257 reflections with $I>2 \sigma(I)$

$\theta_{\max }=32.96^{\circ}$

$$
\begin{aligned}
& h=0 \rightarrow 17 \\
& k=0 \rightarrow 16 \\
& l=-12 \rightarrow 12 \\
& 3 \text { standard reflections } \\
& \quad \text { frequency: } 60 \text { min } \\
& \text { intensity decay: none }
\end{aligned}
$$

\section{Refinement}

Refinement on $F^{2}$

$R\left[F^{2}>2 \sigma\left(F^{2}\right)\right]=0.034$

$w R\left(F^{2}\right)=0.105$

$S=1.080$

3777 reflections

124 parameters

H-atom parameters constrained
Table 1

Selected geometric parameters $\left(\AA,^{\circ}\right)$.

\begin{tabular}{llll}
\hline $\mathrm{P} 1-\mathrm{O} 4$ & $1.4968(7)$ & $\mathrm{N} 3-\mathrm{C} 5$ & $1.461(2)$ \\
$\mathrm{P} 1-\mathrm{O} 3$ & $1.5148(7)$ & $\mathrm{N} 3-\mathrm{C} 4$ & $1.458(2)$ \\
$\mathrm{P} 1-\mathrm{O} 1$ & $1.5629(8)$ & $\mathrm{N} 2-\mathrm{C} 1$ & $1.3447(15)$ \\
$\mathrm{P} 1-\mathrm{O} 2$ & $1.5800(8)$ & $\mathrm{N} 2-\mathrm{C} 3$ & $1.466(2)$ \\
$\mathrm{N} 1-\mathrm{C} 1$ & $1.3198(13)$ & $\mathrm{N} 2-\mathrm{C} 2$ & $1.455(2)$ \\
$\mathrm{N} 3-\mathrm{C} 1$ & $1.3451(14)$ & & \\
& & & \\
$\mathrm{O} 4-\mathrm{P} 1-\mathrm{O} 3$ & $114.78(5)$ & $\mathrm{C} 5-\mathrm{N} 3-\mathrm{C} 4$ & $114.50(12)$ \\
$\mathrm{O} 4-\mathrm{P} 1-\mathrm{O} 1$ & $108.64(5)$ & $\mathrm{C} 1-\mathrm{N} 2-\mathrm{C} 3$ & $121.02(12)$ \\
$\mathrm{O} 3-\mathrm{P} 1-\mathrm{O} 1$ & $109.11(4)$ & $\mathrm{C} 1-\mathrm{N} 2-\mathrm{C} 2$ & $121.27(11)$ \\
$\mathrm{O} 4-\mathrm{P} 1-\mathrm{O} 2$ & $110.24(4)$ & $\mathrm{C} 3-\mathrm{N} 2-\mathrm{C} 2$ & $115.34(12)$ \\
$\mathrm{O} 3-\mathrm{P} 1-\mathrm{O} 2$ & $108.20(5)$ & $\mathrm{N} 1-\mathrm{C} 1-\mathrm{N} 2$ & $120.78(11)$ \\
$\mathrm{O} 1-\mathrm{P} 1-\mathrm{O} 2$ & $105.47(5)$ & $\mathrm{N} 1-\mathrm{C} 1-\mathrm{N} 3$ & $120.22(10)$ \\
$\mathrm{C} 1-\mathrm{N} 3-\mathrm{C} 5$ & $120.91(10)$ & $\mathrm{N} 2-\mathrm{C} 1-\mathrm{N} 3$ & $119.01(10)$ \\
$\mathrm{C} 1-\mathrm{N} 3-\mathrm{C} 4$ & $121.53(12)$ & & \\
\hline
\end{tabular}

Table 2

Hydrogen-bonding geometry $\left(\AA{ }^{\circ},{ }^{\circ}\right)$.

\begin{tabular}{lllll}
\hline$D-\mathrm{H} \cdots A$ & $D-\mathrm{H}$ & $\mathrm{H} \cdots A$ & $D \cdots A$ & $D-\mathrm{H} \cdots A$ \\
\hline $\mathrm{N} 1-\mathrm{H} 1 A \cdots \mathrm{O} 3$ & 0.88 & 2.08 & $2.924(1)$ & 161 \\
$\mathrm{O} 2-\mathrm{H} 2 \cdots \mathrm{O} 3^{\mathrm{i}}$ & 0.84 & 1.79 & $2.616(1)$ & 167 \\
$\mathrm{O} 1-\mathrm{H} 1 \cdots \mathrm{O} 4^{\mathrm{ii}}$ & 0.84 & 1.71 & $2.541(1)$ & 169 \\
$\mathrm{~N} 1-\mathrm{H} 1 B \cdots \mathrm{O} 3^{\text {iii }}$ & 0.88 & 2.07 & $2.894(1)$ & 155 \\
$\mathrm{C} 5-\mathrm{H} 5 B \cdots \mathrm{O} 2^{\text {iii }}$ & 0.98 & 2.56 & $3.521(2)$ & 167 \\
$\mathrm{C} 5-\mathrm{H} 5 A \cdots \mathrm{O} 2^{\text {iv }}$ & 0.98 & 2.67 & $3.607(2)$ & 159 \\
\hline
\end{tabular}

Symmetry codes: (i) $x,-\frac{1}{2}-y, z-\frac{1}{2}$; (ii) $x,-\frac{1}{2}-y, \frac{1}{2}+z$; (iii) $-x,-y, 1-z$; (iv) $x, 1+y, z$.

Data collection and cell refinement: $C A D-4$ Software (EnrafNonius, 1989); data reduction: XRAY76 (Stewart et al., 1976); program(s) used to solve structure: SIR92 (Altomare et al., 1994); program(s) used to refine structure: SHELXL93 (Sheldrick, 1993); molecular graphics: PLATON (Spek, 1994); software used to prepare material for publication: PARST (Nardelli, 1995) and PARSTCIF (Nardelli, 1991).

This work was supported by the Spanish CICYT project PB98-1126. We also acknowledge travel support from Accion Integrada Hispano-Portuguesa HP 1999-0070.

Supplementary data for this paper are available from the IUCr electronic archives (Reference: NA1466). Services for accessing these data are described at the back of the journal.

\section{References}

Adams, J. M. (1977). Acta Cryst. B33, 1513-1515.

Allen, F. H., Kennard, O., Watson, D. G., Brammer, L., Orpen, A. G. \& Taylor, R. (1987). J. Chem. Soc. Perkin Trans. 2, pp. S1-19.

Altomare, A., Cascarano, G., Giacovazzo, C., Guagliardi, A., Burla, M. C., Polidori, G. \& Camalli, M. (1994). J. Appl. Cryst. 27, 435.

Aoki, K., Nagano, K. \& Iitaka, Y. (1971). Acta Cryst. B27, 11-23.

Averbuch-Pouchot, A. T., Durif, A. \& Guitel, J. C. (1988). Acta Cryst. C44, 99 102.

Enraf-Nonius (1989). CAD-4 Software. Version 5.0. Enraf-Nonius, Delft, The Netherlands.

Kotler, Z., Hierle, R., Josse, D., Zyss, J. \& Masse, R. (1992). J. Opt. Soc. Am. B9, 534-547.

Masse, R. \& Zyss, J. (1991). Mol. Eng. 1, 141-152.

Nardelli, M. (1991). PARSTCIF. University of Parma, Italy.

Nardelli, M. (1995). J. Appl. Cryst. 28, 659.

Rafhkovich, L. N. (1991). KDP-Family Single Crystals. Bristol: IOP Publishing. Sheldrick, G. M. (1993). SHELXL93. University of Göttingen, Germany. Spek, A. L. (1994). PLATON. University of Utrecht, The Netherlands.

Stewart, J. M., Machin, P. A., Dickinson, C. W., Ammon, H. L., Heck, H. \& Flack, H. (1976). The XRAY76 System. Technical Report TR-446. Computer Science Center, University of Maryland, USA. 


\section{supporting information}

Acta Cryst. (2000). C56, 888-889 [doi:10.1107/S0108270100005187]

\section{1,1,3,3-Tetramethylguanidinium dihydrogenorthophosphate}

\section{A. Criado, M. J. Diánez, S. Pérez-Garrido, I. M. L. Fernandes, M. Belsley and E. de Matos}

\section{Gomes}

\section{Computing details}

Data collection: CAD-4 Software (Enraf-Nonius, 1989); cell refinement: SET4 (de Boer \& Duissenberg, 1984) and CELDIM (CAD4, Retting, 1989); data reduction: XRAY76 System (Stewart et al., 1976); program(s) used to solve structure: SIR92 (Altomare et al., 1994); program(s) used to refine structure: SHELXL93 (Sheldrick, 1993); molecular graphics: PLATON (Spek, 1994); software used to prepare material for publication: PARST (Nardelli, 1995) and PARSTCIF (Nardelli, 1991).

\section{1,1,3,3-tetramethyl guanidinium phosphate}

Crystal data

$\mathrm{C}_{5} \mathrm{H}_{14} \mathrm{~N}_{3}{ }^{+} \cdot \mathrm{H}_{2} \mathrm{PO}_{4}$

$M_{r}=213.18$

Monoclinic, $P 2_{1} / c$

$a=11.225(3) \AA$

$b=10.951(1) \AA$

$c=8.430(2) \AA$

$\beta=103.50(1)^{\circ}$

$V=1007.6(4) \AA^{3}$

$Z=4$

$F(000)=456$

\section{Data collection}

Enraf-Nonius CAD4 diffractometer

Radiation source: fine-focus sealed tube Graphite monochromator $\omega-2 \theta$ scans

3777 measured reflections 3777 independent reflections

3257 reflections with $I>2 \sigma(I)$

\section{Refinement}

Refinement on $F^{2}$

Least-squares matrix: full

$R\left[F^{2}>2 \sigma\left(F^{2}\right)\right]=0.034$

$w R\left(F^{2}\right)=0.105$

$S=1.08$

3777 reflections

$$
\begin{aligned}
& D_{\mathrm{x}}=1.405 \mathrm{Mg} \mathrm{m}^{-3} \\
& D_{\mathrm{m}}=1.40 \mathrm{Mg} \mathrm{m}^{-3} \\
& D_{\mathrm{m}} \text { measured by flotation in bromobenzene and } \\
& \text { acetone } \\
& \text { Melting point: } 493 \mathrm{~K} \\
& \text { Mo } K \alpha \text { radiation, } \lambda=0.71069 \AA \\
& \text { Cell parameters from } 25 \text { reflections } \\
& \theta=7-12^{\circ} \\
& \mu=0.26 \mathrm{~mm}^{-1} \\
& T=293 \mathrm{~K} \\
& \text { Prism, colourless } \\
& 0.80 \times 0.50 \times 0.40 \mathrm{~mm}
\end{aligned}
$$

$$
\begin{aligned}
& R_{\text {int }}=0.000 \\
& \theta_{\max }=33.0^{\circ}, \theta_{\min }=2.6^{\circ} \\
& h=0 \rightarrow 17 \\
& k=0 \rightarrow 16 \\
& l=-12 \rightarrow 12 \\
& 3 \text { standard reflections every } 60 \text { min min } \\
& \text { intensity decay: none }
\end{aligned}
$$

\footnotetext{
124 parameters

0 restraints

Primary atom site location: structure-invariant direct methods

Secondary atom site location: difference Fourier map
} 
Hydrogen site location: inferred from neighbouring sites

H-atom parameters constrained

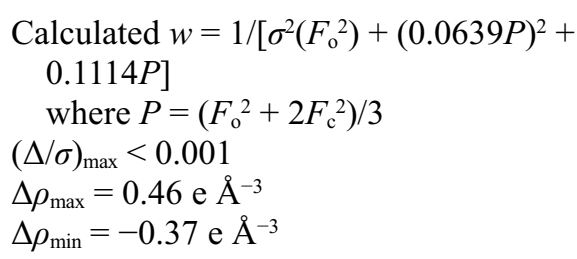

Special details

Geometry. All e.s.d.'s (except the e.s.d. in the dihedral angle between two 1.s. planes) are estimated using the full covariance matrix. The cell e.s.d.'s are taken into account individually in the estimation of e.s.d.'s in distances, angles and torsion angles; correlations between e.s.d.'s in cell parameters are only used when they are defined by crystal symmetry. An approximate (isotropic) treatment of cell e.s.d.'s is used for estimating e.s.d.'s involving l.s. planes.

Refinement. Refinement on $F^{2}$ for ALL reflections except for 0 with very negative $F^{2}$ or flagged by the user for potential systematic errors. Weighted $R$-factors $w R$ and all goodnesses of fit $S$ are based on $F^{2}$, conventional $R$-factors $R$ are based on $F$, with $F$ set to zero for negative $F^{2}$. The observed criterion of $F^{2}>\sigma\left(F^{2}\right)$ is used only for calculating R_factor_obs etc. and is not relevant to the choice of reflections for refinement. $R$-factors based on $F^{2}$ are statistically about twice as large as those based on $F$, and $R$ - factors based on ALL data will be even larger.

Fractional atomic coordinates and isotropic or equivalent isotropic displacement parameters $\left(\AA^{2}\right)$

\begin{tabular}{|c|c|c|c|c|}
\hline & $x$ & $y$ & $z$ & $U_{\text {iso }} * / U_{\text {eq }}$ \\
\hline $\mathrm{P} 1$ & $0.21200(2)$ & -0.22099 (2) & $0.44607(3)$ & $0.02297(8)$ \\
\hline $\mathrm{O} 4$ & $0.26662(8)$ & $-0.14310(7)$ & $0.33544(9)$ & $0.0318(2)$ \\
\hline $\mathrm{O} 3$ & $0.12944(7)$ & $-0.15363(7)$ & $0.53532(9)$ & $0.0296(2)$ \\
\hline $\mathrm{O} 2$ & $0.13625(9)$ & $-0.32927(8)$ & $0.34687(10)$ & $0.0384(2)$ \\
\hline $\mathrm{H} 2$ & 0.1421 & -0.3263 & 0.2494 & $0.058^{*}$ \\
\hline O1 & $0.31819(7)$ & $-0.28321(8)$ & $0.57362(10)$ & $0.0348(2)$ \\
\hline H1 & 0.2919 & -0.3067 & 0.6540 & $0.052 *$ \\
\hline N1 & $0.13166(8)$ & $0.11300(8)$ & $0.51765(13)$ & $0.0358(2)$ \\
\hline $\mathrm{H} 1 \mathrm{~A}$ & 0.14840 & 0.03517 & 0.53720 & $0.043 *$ \\
\hline H1B & 0.05591 & 0.13620 & 0.47485 & $0.043^{*}$ \\
\hline N3 & $0.19506(10)$ & $0.31397(9)$ & $0.52252(13)$ & $0.0386(2)$ \\
\hline $\mathrm{N} 2$ & $0.33639(9)$ & $0.16108(10)$ & $0.61799(14)$ & $0.0415(2)$ \\
\hline $\mathrm{C} 1$ & $0.22015(10)$ & $0.19494(10)$ & $0.55254(13)$ & 0.0309 (2) \\
\hline C5 & $0.08049(12)$ & $0.35246(12)$ & $0.4126(2)$ & 0.0459 (3) \\
\hline $\mathrm{H} 5 \mathrm{~A}$ & 0.0924 & 0.4317 & 0.3645 & $0.069^{*}$ \\
\hline H5B & 0.0166 & 0.3597 & 0.4739 & $0.069^{*}$ \\
\hline $\mathrm{H} 5 \mathrm{C}$ & 0.0556 & 0.2918 & 0.3257 & $0.069 *$ \\
\hline $\mathrm{C} 2$ & $0.36472(13)$ & $0.04187(13)$ & $0.6937(2)$ & $0.0530(4)$ \\
\hline $\mathrm{H} 2 \mathrm{~A}$ & 0.4411 & 0.0469 & 0.7782 & $0.080^{*}$ \\
\hline $\mathrm{H} 2 \mathrm{~B}$ & 0.3742 & -0.0177 & 0.6108 & $0.080 *$ \\
\hline $\mathrm{H} 2 \mathrm{C}$ & 0.2979 & 0.0162 & 0.7430 & $0.080^{*}$ \\
\hline $\mathrm{C} 4$ & $0.2563(2)$ & $0.40878(13)$ & $0.6335(2)$ & $0.0626(4)$ \\
\hline $\mathrm{H} 4 \mathrm{~A}$ & 0.1968 & 0.4485 & 0.6851 & $0.094 *$ \\
\hline H4B & 0.2915 & 0.4695 & 0.5723 & $0.094 *$ \\
\hline $\mathrm{H} 4 \mathrm{C}$ & 0.3216 & 0.3721 & 0.7177 & $0.094 *$ \\
\hline C3 & $0.43961(13)$ & $0.2244(2)$ & $0.5750(2)$ & $0.0630(5)$ \\
\hline $\mathrm{H} 3 \mathrm{~A}$ & 0.4925 & 0.1646 & 0.5386 & $0.094^{*}$ \\
\hline H3B & 0.4867 & 0.2681 & 0.6707 & $0.094 *$ \\
\hline $\mathrm{H} 3 \mathrm{C}$ & 0.40852 & 0.2828 & 0.4870 & $0.094 *$ \\
\hline
\end{tabular}


supporting information

Atomic displacement parameters $\left(\AA^{2}\right)$

\begin{tabular}{lllllll}
\hline & $U^{11}$ & $U^{22}$ & $U^{33}$ & $U^{12}$ & $U^{13}$ & $U^{23}$ \\
\hline P1 & $0.02584(12)$ & $0.02308(12)$ & $0.02056(11)$ & $0.00027(7)$ & $0.00656(8)$ & $0.00083(7)$ \\
O4 & $0.0431(4)$ & $0.0283(3)$ & $0.0264(3)$ & $-0.0075(3)$ & $0.0125(3)$ & $0.0006(3)$ \\
O3 & $0.0303(3)$ & $0.0317(3)$ & $0.0277(3)$ & $0.0081(3)$ & $0.0087(3)$ & $0.0015(3)$ \\
O2 & $0.0539(5)$ & $0.0356(4)$ & $0.0279(3)$ & $-0.0179(4)$ & $0.0137(3)$ & $-0.0048(3)$ \\
O1 & $0.0294(3)$ & $0.0464(5)$ & $0.0301(3)$ & $0.0121(3)$ & $0.0100(3)$ & $0.0092(3)$ \\
N1 & $0.0266(4)$ & $0.0261(4)$ & $0.0535(6)$ & $0.0007(3)$ & $0.0072(4)$ & $-0.0021(4)$ \\
N3 & $0.0430(5)$ & $0.0271(4)$ & $0.0458(5)$ & $-0.0043(4)$ & $0.0105(4)$ & $-0.0041(4)$ \\
N2 & $0.0282(4)$ & $0.0438(5)$ & $0.0496(6)$ & $-0.0022(4)$ & $0.0032(4)$ & $-0.0042(4)$ \\
C1 & $0.0300(4)$ & $0.0297(4)$ & $0.0339(5)$ & $-0.0012(3)$ & $0.0093(4)$ & $-0.0058(4)$ \\
C5 & $0.0426(6)$ & $0.0347(6)$ & $0.0626(8)$ & $0.0028(5)$ & $0.0168(6)$ & $0.0122(5)$ \\
C2 & $0.0417(6)$ & $0.0429(7)$ & $0.0653(9)$ & $0.0081(5)$ & $-0.0059(6)$ & $-0.0054(6)$ \\
C4 & $0.0952(13)$ & $0.0332(6)$ & $0.0558(8)$ & $-0.0164(7)$ & $0.0105(8)$ & $-0.0130(6)$ \\
C3 & $0.0298(6)$ & $0.0859(13)$ & $0.0708(10)$ & $-0.0132(7)$ & $0.0071(6)$ & $0.0034(9)$ \\
& & & & & &
\end{tabular}

Geometric parameters $\left(A,{ }^{o}\right)$

\begin{tabular}{|c|c|c|c|}
\hline $\mathrm{P} 1-\mathrm{O} 4$ & $1.4968(7)$ & $\mathrm{N} 2-\mathrm{C} 2$ & $1.455(2)$ \\
\hline $\mathrm{P} 1-\mathrm{O} 3$ & $1.5148(7)$ & $\mathrm{C} 5-\mathrm{H} 5 \mathrm{~A}$ & 0.98 \\
\hline $\mathrm{P} 1-\mathrm{O} 1$ & $1.5629(8)$ & C5-H5B & 0.98 \\
\hline $\mathrm{P} 1-\mathrm{O} 2$ & $1.5800(8)$ & $\mathrm{C} 5-\mathrm{H} 5 \mathrm{C}$ & 0.98 \\
\hline $\mathrm{O} 2-\mathrm{H} 2$ & 0.84 & $\mathrm{C} 2-\mathrm{H} 2 \mathrm{~A}$ & 0.98 \\
\hline $\mathrm{O} 1-\mathrm{H} 1$ & 0.84 & $\mathrm{C} 2-\mathrm{H} 2 \mathrm{~B}$ & 0.98 \\
\hline $\mathrm{N} 1-\mathrm{C} 1$ & $1.3198(13)$ & $\mathrm{C} 2-\mathrm{H} 2 \mathrm{C}$ & 0.98 \\
\hline $\mathrm{N} 1-\mathrm{H} 1 \mathrm{~A}$ & 0.88 & $\mathrm{C} 4-\mathrm{H} 4 \mathrm{~A}$ & 0.98 \\
\hline $\mathrm{N} 1-\mathrm{H} 1 \mathrm{~B}$ & 0.88 & $\mathrm{C} 4-\mathrm{H} 4 \mathrm{~B}$ & 0.98 \\
\hline $\mathrm{N} 3-\mathrm{C} 1$ & $1.3451(14)$ & $\mathrm{C} 4-\mathrm{H} 4 \mathrm{C}$ & 0.98 \\
\hline $\mathrm{N} 3-\mathrm{C} 5$ & $1.461(2)$ & $\mathrm{C} 3-\mathrm{H} 3 \mathrm{~A}$ & 0.98 \\
\hline $\mathrm{N} 3-\mathrm{C} 4$ & $1.458(2)$ & $\mathrm{C} 3-\mathrm{H} 3 \mathrm{~B}$ & 0.98 \\
\hline $\mathrm{N} 2-\mathrm{C} 1$ & $1.3447(15)$ & $\mathrm{C} 3-\mathrm{H} 3 \mathrm{C}$ & 0.98 \\
\hline $\mathrm{N} 2-\mathrm{C} 3$ & $1.466(2)$ & & \\
\hline $\mathrm{O} 4-\mathrm{P} 1-\mathrm{O} 3$ & $114.78(5)$ & $\mathrm{H} 5 \mathrm{~A}-\mathrm{C} 5-\mathrm{H} 5 \mathrm{~B}$ & 109.5 \\
\hline $\mathrm{O} 4-\mathrm{P} 1-\mathrm{O} 1$ & $108.64(5)$ & $\mathrm{N} 3-\mathrm{C} 5-\mathrm{H} 5 \mathrm{C}$ & 109.47 \\
\hline $\mathrm{O} 3-\mathrm{P} 1-\mathrm{O} 1$ & $109.11(4)$ & $\mathrm{H} 5 \mathrm{~A}-\mathrm{C} 5-\mathrm{H} 5 \mathrm{C}$ & 109.5 \\
\hline $\mathrm{O} 4-\mathrm{P} 1-\mathrm{O} 2$ & $110.24(4)$ & $\mathrm{H} 5 \mathrm{~B}-\mathrm{C} 5-\mathrm{H} 5 \mathrm{C}$ & 109.5 \\
\hline $\mathrm{O} 3-\mathrm{P} 1-\mathrm{O} 2$ & $108.20(5)$ & $\mathrm{N} 2-\mathrm{C} 2-\mathrm{H} 2 \mathrm{~A}$ & 109.47 \\
\hline $\mathrm{O} 1-\mathrm{P} 1-\mathrm{O} 2$ & $105.47(5)$ & $\mathrm{N} 2-\mathrm{C} 2-\mathrm{H} 2 \mathrm{~B}$ & 109.47 \\
\hline $\mathrm{P} 1-\mathrm{O} 2-\mathrm{H} 2$ & 109.47 & $\mathrm{H} 2 \mathrm{~A}-\mathrm{C} 2-\mathrm{H} 2 \mathrm{~B}$ & 109.5 \\
\hline $\mathrm{P} 1-\mathrm{O} 1-\mathrm{H} 1$ & 109.47 & $\mathrm{~N} 2-\mathrm{C} 2-\mathrm{H} 2 \mathrm{C}$ & 109.47 \\
\hline $\mathrm{C} 1-\mathrm{N} 1-\mathrm{H} 1 \mathrm{~A}$ & 120.00 & $\mathrm{H} 2 \mathrm{~A}-\mathrm{C} 2-\mathrm{H} 2 \mathrm{C}$ & 109.5 \\
\hline $\mathrm{C} 1-\mathrm{N} 1-\mathrm{H} 1 \mathrm{~B}$ & 120.00 & $\mathrm{H} 2 \mathrm{~B}-\mathrm{C} 2-\mathrm{H} 2 \mathrm{C}$ & 109.5 \\
\hline $\mathrm{H} 1 \mathrm{~A}-\mathrm{N} 1-\mathrm{H} 1 \mathrm{~B}$ & 120.0 & $\mathrm{~N} 3-\mathrm{C} 4-\mathrm{H} 4 \mathrm{~A}$ & 109.47 \\
\hline $\mathrm{C} 1-\mathrm{N} 3-\mathrm{C} 5$ & $120.91(10)$ & $\mathrm{N} 3-\mathrm{C} 4-\mathrm{H} 4 \mathrm{~B}$ & 109.47 \\
\hline $\mathrm{C} 1-\mathrm{N} 3-\mathrm{C} 4$ & $121.53(12)$ & $\mathrm{H} 4 \mathrm{~A}-\mathrm{C} 4-\mathrm{H} 4 \mathrm{~B}$ & 109.5 \\
\hline $\mathrm{C} 5-\mathrm{N} 3-\mathrm{C} 4$ & $114.50(12)$ & $\mathrm{N} 3-\mathrm{C} 4-\mathrm{H} 4 \mathrm{C}$ & 109.47 \\
\hline
\end{tabular}


$\mathrm{C} 1-\mathrm{N} 2-\mathrm{C} 3$

$\mathrm{C} 1-\mathrm{N} 2-\mathrm{C} 2$

$\mathrm{C} 3-\mathrm{N} 2-\mathrm{C} 2$

$\mathrm{N} 1-\mathrm{C} 1-\mathrm{N} 2$

$\mathrm{N} 1-\mathrm{C} 1-\mathrm{N} 3$

$\mathrm{N} 2-\mathrm{C} 1-\mathrm{N} 3$

$\mathrm{N} 3-\mathrm{C} 5-\mathrm{H} 5 \mathrm{~A}$

N3- C5-H5B
$121.02(12)$

$121.27(11)$

$115.34(12)$

$120.78(11)$

$120.22(10)$

$119.01(10)$

109.47

109.47
$\mathrm{H} 4 \mathrm{~A}-\mathrm{C} 4-\mathrm{H} 4 \mathrm{C}$
$\mathrm{H} 4 \mathrm{~B}-\mathrm{C} 4-\mathrm{H} 4 \mathrm{C}$
$\mathrm{N} 2-\mathrm{C} 3-\mathrm{H} 3 \mathrm{~A}$
$\mathrm{~N} 2-\mathrm{C} 3-\mathrm{H} 3 \mathrm{~B}$
$\mathrm{H} 3 \mathrm{~A}-\mathrm{C} 3-\mathrm{H} 3 \mathrm{~B}$
$\mathrm{~N} 2-\mathrm{C} 3-\mathrm{H} 3 \mathrm{C}$
$\mathrm{H} 3 \mathrm{~A}-\mathrm{C} 3-\mathrm{H} 3 \mathrm{C}$
$\mathrm{H} 3 \mathrm{~B}-\mathrm{C} 3-\mathrm{H} 3 \mathrm{C}$

109.5

109.5

109.47

109.47

109.5

109.47

109.5

109.5

Hydrogen-bond geometry $\left(\AA,{ }^{\circ}\right)$

\begin{tabular}{lllll}
\hline$D-\mathrm{H}^{\prime} \cdots$ & $D-\mathrm{H}$ & $\mathrm{H} \cdots A$ & $D \cdots A$ & $D-\mathrm{H}^{\cdots} A$ \\
\hline $\mathrm{N} 1-\mathrm{H} 1 A^{\cdots} \cdots \mathrm{O} 3$ & 0.88 & 2.08 & $2.924(1)$ & 161 \\
$\mathrm{O} 2-\mathrm{H} 2 \cdots \mathrm{O} 3^{\mathrm{i}}$ & 0.84 & 1.79 & $2.616(1)$ & 167 \\
$\mathrm{O} 1-\mathrm{H} 1 \cdots \mathrm{O} 4^{\mathrm{ii}}$ & 0.84 & 1.71 & $2.541(1)$ & 169 \\
$\mathrm{~N} 1-\mathrm{H} 1 B \cdots \mathrm{O} 3^{\text {iii }}$ & 0.88 & 2.07 & $2.894(1)$ & 155 \\
$\mathrm{C} 5-\mathrm{H} 5 B \cdots \mathrm{O} 2^{\text {iii }}$ & 0.98 & 2.56 & $3.521(2)$ & 167 \\
$\mathrm{C} 5-\mathrm{H} 5 A \cdots \mathrm{O} 2^{\text {iv }}$ & 0.98 & 2.67 & $3.607(2)$ & 159 \\
\hline
\end{tabular}

Symmetry codes: (i) $x,-y-1 / 2, z-1 / 2$; (ii) $x,-y-1 / 2, z+1 / 2$; (iii) $-x,-y,-z+1$; (iv) $x, y+1, z$. 